\title{
Recombinant human erythropoietin separation using a cation-exchange multimodal adsorbent
}

\author{
Marta Ostrihoňová, Jana Adamíková, Tomáš Molnár, \\ Monika Antošová, Milan Polakovič \\ Department of Chemical and Biochemical Engineering, Institute of Chemical and Environmental Engineering, \\ Faculty of Chemical and Food Technology, Slovak University of Technology, \\ Radlinského 9, 81237 Bratislava, Bratislava, Slovakia \\ milan.polakovic@stuba.sk
}

\begin{abstract}
This work deals with the capture of human recombinant erythropoietin (rhEPO) from a mixture of proteins in a concentrated postcultivation supernatant. Cation-exchange multimodal adsorbent Capto MMC ImpRes was selected as potential chromatographic separation material. Its equilibrium properties were investigated in batch adsorption experiments. The effect of $\mathrm{pH}$ in the range of 5.5-7.5 and $\mathrm{NaCl}$ concentration in the range of $0-300 \mathrm{mM}$ on the adsorption of rhEPO and contaminant proteins was examined. Optimal conditions found in these equilibrium experiments were applied to rhEPO adsorption in a chromatographic column. Several experiments were carried out at different elution conditions to optimize the rhEPO yield and selectivity.
\end{abstract}

Keywords: erythropoietin, multimodal adsorbent, selectivity, elution chromatography

\section{Introduction}

Erythropoietin is a human hormone involved in the formation of red blood cells. Recombinant human erythropoietin (rhEPO) is a commercially available drug used to treat anaemia and other diseases. rhEPO produced by mammalian cells is secreted into the growth medium and subsequently isolated and purified to the required purity.

During purification, all foreign proteins, viruses and toxins have to be removed because they can cause an undesirable immune response. Different chromatographic methods are used for rhEPO purification (Adamíková et al., 2019). Size-exclusion, ion-exchange, hydrophobic, affinity and multimodal interactions between protein molecules and adsorbent are the most commonly applied in rhEPO purification.

Multimodal chromatography is the novel process separation mode which was originally developed for monoclonal antibodies purification. Multimodal adsorbents contain several different functional groups, which allows simultaneous binding of proteins through different interactions. Multimodal adsorbents offer different selectivity compared to classical ion exchangers and have larger operating range in terms of adsorption buffer conductivity (Holstein et al.). Nowadays, there are several applications of multimodal adsorbents for recombinant proteins purification, but applications for rhEPO purification are rare (Koticha and Chaudhari, 2011; Bandi et al., 2017). Our main goal was to determine optimal conditions of rhEPO binding to adsorbent Capto MMC ImpRes from a real mixture and to find appropriate elution of bound proteins.

\section{Materials and methods}

\section{Adsorbent}

Multimodal adsorbent Capto MMC ImpRes (GE Healthcare, Uppsala) has a matrix consisting of agarose chains. Its ligand is shown in Fig. 1. This ligand can provide ionic interactions (1), hydrogen bonds (2), hydrophobic interactions (3) and thiophilic bridges (4) with molecules.

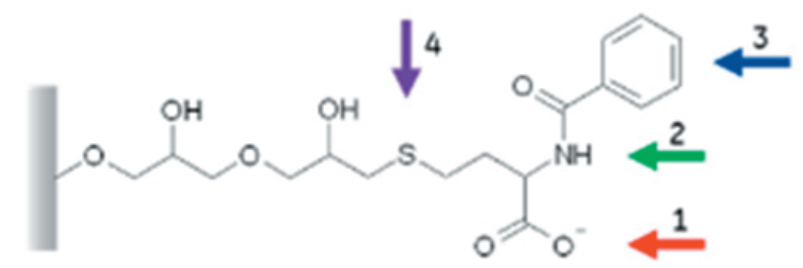

Fig. 1. Structural formula of ligand Capto MMC ImpRes.

\section{rhEPO post-culture supernatants}

Two different batches of a post-culture supernatant containing rhEPO produced by human embryonic kidney cells 293 were used (Molnár et al., 2019). The first one was concentrated and desalted using different membrane filtration processes (Molnár et al., 2019). The final rhEPO concentration and total protein concentration were $0.05 \mathrm{~g} / 1$ and $0.8 \mathrm{~g} / \mathrm{l}$, respectively. This protein solution was used in batch equilibrium experiments and separation runs $\mathrm{S} 1-$ S5 (Table 1). The second batch contained $0.08 \mathrm{~g} / 1$ 
of rhEPO and $2.1 \mathrm{~g} / 1$ of total proteins and was used in the separation run S6 (Table 1). This postculture supernatant was not treated via membrane processes. Only $\mathrm{pH}$ was set by concentrated $\mathrm{HCl}$ and $300 \mathrm{mM} \mathrm{NaCl}$ was added into the supernatant before adsorption.

\section{Batch adsorption}

Adsorbent in the amount of $25 \mathrm{mg}$ was placed into a syringe filter, washed three times with $1 \mathrm{ml}$ of distilled water and five times with $1 \mathrm{ml}$ of $50 \mathrm{mM}$ citrate phosphate buffer. The equilibrated adsorbent was suspended in $1 \mathrm{ml}$ of adjusted rhEPO-containing solution and the suspension was stirred for 20 hours at the frequency of $80 \mathrm{rpm}$ using a rotary shaker. The content of rhEPO and total proteins in the solution before and after the adsorption were determined and the adsorbed rhEPO/total protein amount was calculated from the material balance.

\section{Column separation}

System ÄKTA FPLC ${ }^{\mathrm{TM}}$ (GE Healthcare) was used for rhEPO separation from the rhEPO concentrate. Chromatographic column Tricorn (GE Healthcare) was filled with the adsorbent Capto MMC ImpRes. The column adsorbent volume was $1.198 \mathrm{ml}$ when the bed height and diameter were $6.1 \mathrm{~cm}$ and $0.5 \mathrm{~cm}$, respectively.

Tab. 1. Eluent composition in separation runs.

\begin{tabular}{ll}
\hline Run & Eluent $^{*}$ \\
\hline S1 & Shock-wave gradients: $\mathrm{B} 1 \rightarrow \mathrm{B} 2 \rightarrow \mathrm{B} 6$ \\
S2 & Shock-wave gradients: $\mathrm{B} 3 \rightarrow \mathrm{B} 4 \rightarrow \mathrm{B} 6$ \\
S3 & Shock-wave gradients: $\mathrm{B} 5 \rightarrow \mathrm{B} 6$ \\
S4 & Linear gradient B1/B5 $\rightarrow$ shock-wave gradient B6 \\
S5 & Shock-wave gradients: B $\rightarrow \mathrm{B} 8 \rightarrow \mathrm{B} 6$ \\
S6 & Shock-wave gradients: $\mathrm{B} 3 \rightarrow \mathrm{B} 4 \rightarrow \mathrm{B} 6$
\end{tabular}

${ }^{*}$ Symbols B1-B8 refer to different buffers specified in Table 2.

Tab. 2. Elution buffers.

\begin{tabular}{ll}
\hline Buffer code & Buffer type* $^{*}$ \\
\hline B1 & Tris, pH 7 \\
B2 & Tris/1 M NaCl, pH 7 \\
B3 & Tris, pH 8 \\
B4 & Tris/1 M NaCl, pH 8 \\
B5 & Tris, pH 9 \\
B6 & Tris/1 M NaCl, pH 9 \\
B7 & CF/2 M NaCl, pH 5.5 \\
B8 & CF/2 M NaCl/2 M urea, pH 5.5 \\
\hline
\end{tabular}

"Tris - $50 \mathrm{mM}$ Tris- $\mathrm{HCl}$ buffer, CF $-50 \mathrm{mM}$ citratephosphate buffer.
The adsorbent was first equilibrated with $30 \mathrm{ml}$ of the $50 \mathrm{mM}$ citrate-phosphate buffer containing $300 \mathrm{mM} \mathrm{NaCl}$ at $\mathrm{pH} 5.5$. The feed $\mathrm{pH}$ and composition were adjusted to $\mathrm{pH} 5.5$ and $\mathrm{NaCl}$ concentration of $300 \mathrm{mM}$. The feed volume was either $9 \mathrm{ml}$ (runs $\mathrm{S} 1-\mathrm{S} 5$ ) or $13 \mathrm{ml}$ (run S6) and the feed flow rate was $0.5 \mathrm{ml} / \mathrm{min}$. After the feed loading was finished, the bed was washed with $3.6 \mathrm{ml}$ of the adsorption buffer. Several ways of gradient elution to recover rhEPO from the column were tested (Table 1). The eluent composition was adjusted using different buffers labelled B1-B8 (Table 2). The elution flow rate was $0.5 \mathrm{ml} / \mathrm{min}$ and the UV and conductivity signals were monitored during the whole experiment. Elution fractions were collected and the rhEPO/total protein concentration was determined.

\section{Protein assays}

\section{BCA method}

Protein concentration in batch equilibrium experiments was determined using the BCA method. Working solution was prepared by mixing bicinchoninic acid (BCA) from Sigma-Aldrich (St. Louis, USA) with a $4 \% \mathrm{CuSO}_{4}$ solution in the ratio of 50 : 1. First, a $25 \mu \mathrm{l}$ sample was pipetted into a microplate well. Using a multichannel pipette, $200 \mu \mathrm{l}$ of the working solution was added to the sample. The microplate was incubated for 30 minutes at the temperature of $37^{\circ} \mathrm{C}$. The sample absorbance was measured at the wavelength of $562 \mathrm{~nm}$. Bovine serum albumin (BSA) in the concentration range of 0.05-1 g/l was used for calibration.

\section{Bradford method}

Protein concentration in elution chromatography samples was determined using the Bradford method due to the interference of Tris-HCl buffer with the $\mathrm{BCA}$ reagent. The Bradford agent is a mixture of $0.01 \%$ Coomassie Brilliant Blue G-250, $4.7 \%$ ethanol, and $8.5 \%$ phosphoric acid in deionized water. It was filtered before use. Then, $20 \mu \mathrm{l}$ of the analysed sample and $1500 \mu \mathrm{l}$ of the Bradford agent were pipetted into test tubes. After 10-15 minutes, absorbance was measured at two wavelengths, 590 and $450 \mathrm{~nm}$. BSA solution in the concentration range of $0.05-2 \mathrm{~g} / 1$ was used for calibration.

\section{ELISA method}

rhEPO concentration was determined using a commercial kit of sandwich ELISA method (SigmaAldrich). The sample volume of $100 \mu \mathrm{l}$ was transferred into a microplate well which was incubated for 2.5 hours at the temperature of $25^{\circ} \mathrm{C}$. It was then washed four times with $300 \mu$ l of wash solution. After adding $100 \mu \mathrm{l}$ of biotinylated detection antibody into 
each well, the well plate was incubated for one hour at room temperature. The wells were again washed four times as before. A streptavidin-HRP solution $(100 \mu \mathrm{l})$ was added into each well and the well plate was incubated for 45 minutes at gentle shaking. The washing was repeated again. Finally, $100 \mu \mathrm{l}$ of ELISA colorimetric reagent TMB were added into each well and the well plate was incubated for 30 minutes in dark at gentle shaking. To stop the reaction, $50 \mu \mathrm{l}$ of $0.2 \mathrm{M}$ sulphuric acid were added and the absorbance at $450 \mathrm{~nm}$ was measured.

In run $\mathrm{S6}$, a recently developed indirect ELISA method was used (Molnár et al., 2019).

\section{Results and discussion}

Equilibrium experiments for the determination of optimal rhEPO adsorption conditions were done in the $\mathrm{pH}$ range of 5.5-7.5 and $\mathrm{NaCl}$ concentration range from 0 to $300 \mathrm{mM}$. Equilibrium adsorbed amounts of rhEPO, $q_{\mathrm{EPO}}$, and total proteins, $q_{\text {Prot }}$, are shown in Figs. 2a and 2b. It is evident that higher $\mathrm{pH}$ has negative influence on the adsorption of both rhEPO and total proteins. On the other hand, higher salt concentration promotes rhEPO binding in spite of lower binding of total proteins. This effect is advantageous for selective separation already in the adsorption phase. Fig. 2a shows that the highest amount of rhEPO was adsorbed at $\mathrm{pH}$ 5.5 and $300 \mathrm{mM} \mathrm{NaCl}$. Therefore, these conditions were chosen for chromatography feed.

Efficiency of rhEPO separation was examined in six elution chromatography runs (Fig. 3). For efficient elution, desorption by $1 \mathrm{M} \mathrm{NaCl}$ was first examined in runs $\mathrm{S} 1-\mathrm{S} 3$. Different $\mathrm{pH}$ was also applied in runs $\mathrm{S} 1-\mathrm{S} 3$. Each of these runs started with feeding a salt-free eluent. The NaCl concentration was later increased to $1 \mathrm{M}$ in a shock-wave gradient. In the final elution phase, a shock-wave gradient increase of $\mathrm{pH}$ from 7 (run S1) or 8 (run S2) to the value of 9 was applied. Figs. 3a-3c show abrupt increase of outlet protein concentration demonstrated by the

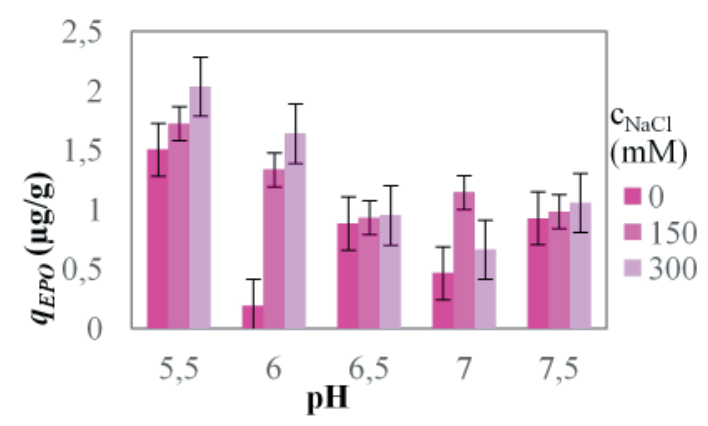

a) increase of UV signal after the application of each gradient change. Numbered peaks denote each elution phase.

These results demonstrate a synergistic effect of $\mathrm{pH}$ and $\mathrm{NaCl}$ concentration on protein recovery. The existence of several elution peaks implies strong protein binding albeit not so strong as in case of multimodal anion exchanger applied to the separation of this product (Kurák et al., 2019). A significant fraction of adsorbed proteins was eluted using salt-free eluents (peak 1 in Figs. 3a-3c). The protein yield increased from $23 \%$ at pH 7 to $50 \%$ at pH 8 and finally to $74 \%$ at pH 9. The application of $1 \mathrm{M} \mathrm{NaCl}$ in the second elution phase led then to almost complete protein recovery. Only in run $\mathrm{S} 1$, $6 \%$ of proteins were recovered in the third phase. Unfortunately, sensitivity of the ELISA method allowed only semi-quantitative estimation of the eluted rhEPO. This was much higher in the second fraction than in the first one in runs $\mathrm{S} 1$ and $\mathrm{S} 2$. In run S3, approximately equal amounts of rhEPO were detected in both fractions.

Following these results, a linear $\mathrm{pH}$ gradient from 7 to 9 and salt-free eluent were applied in the first phase of run S4. Fig. 3d shows that no positive effect was achieved and the separation performance was very similar as in run S1. Considering the distribution of rhEPO and impurity proteins in individual fractions in runs $\mathrm{S} 1-\mathrm{S} 4$, the best selectivity of rhEPO separation was achieved in run S2 carried out at $\mathrm{pH} 8$.

Fig. 3e presents the results of run $\mathrm{S} 5$ which was designed at the same $\mathrm{pH}$ in both the loading and the elution phases. An eluent with stronger desorption effect had to be applied from the beginning of the elution. It is clear that neither $2 \mathrm{M} \mathrm{NaCl}$ nor $2 \mathrm{M} \mathrm{NaCl} / 2 \mathrm{M}$ urea were efficient enough. Complete protein desorption was achieved only when $\mathrm{pH}$ was increased to 9 in the final elution phase.

Fig. 3f presents the results of run $\mathrm{S} 6$ in which the same process conditions were used as in run $\mathrm{S} 2 \mathrm{ex}-$

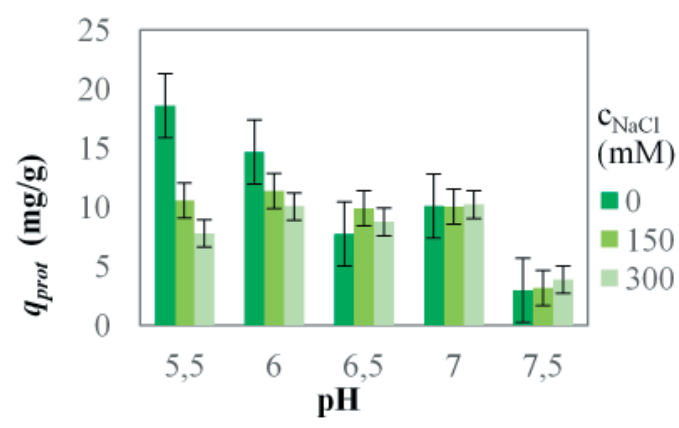

b)

Fig. 2. $\mathrm{pH}$ and $\mathrm{NaCl}$ concentration influence on adsorbed amount of a) rhEPO and b) total proteins. 

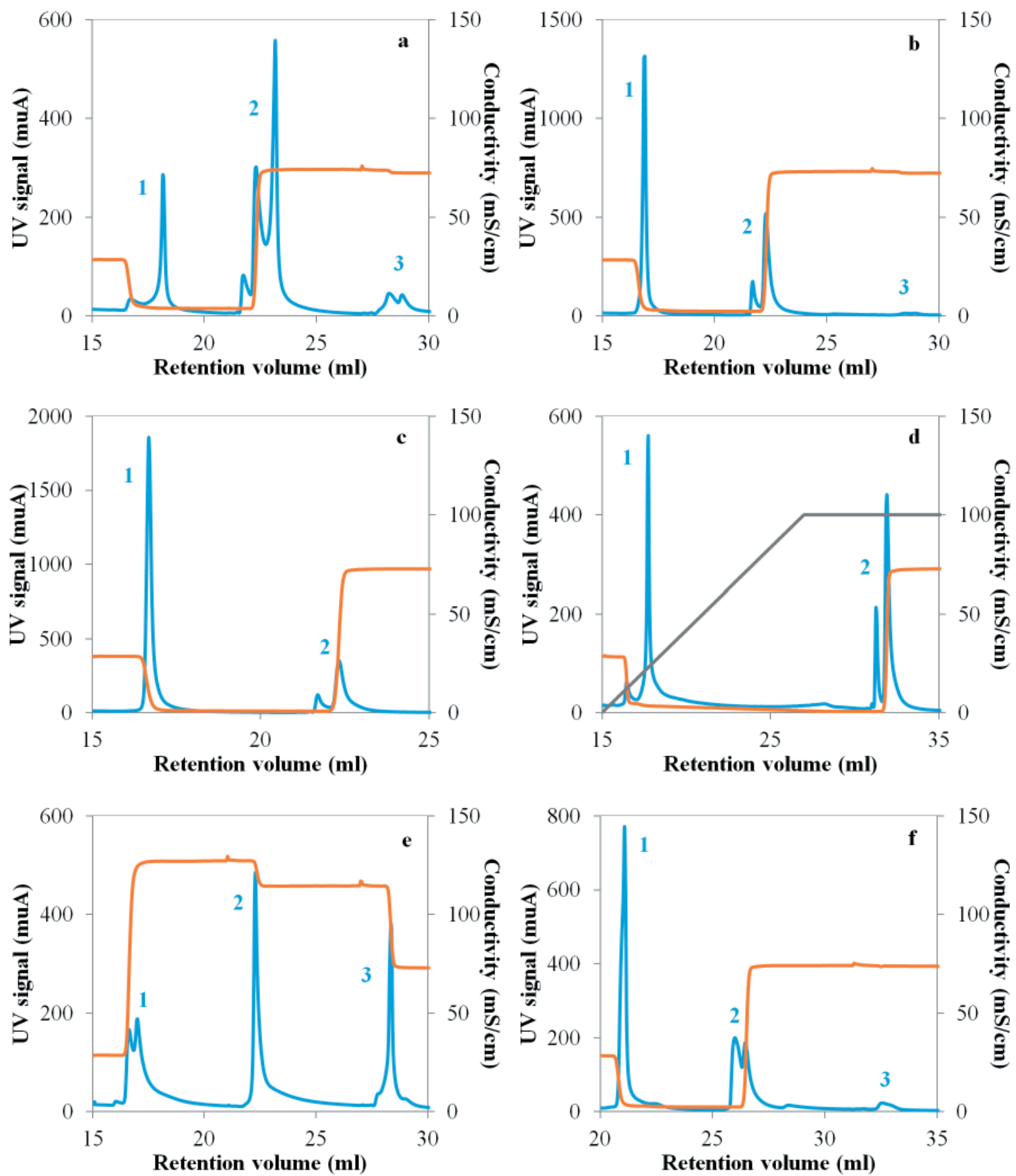

Fig. 3. Chromatograms of individual separation runs: a) S1, b) S2, c) S3, d) S4, e) S5, and f) S6. Blue and orange lines represent UV and conductivity signals, respectively. Peak numbers denote individual elution phases.

cept for a higher feed volume. Since a product from a different cultivation batch was used in this run, the rhEPO and protein loads were more than twice higher. Figs. 3b and 3f show that the chromatograms of runs S2 and S6 are very similar. The protein recovery yields in both phases were almost identical again. Due to a higher rhEPO feed concentration in run $\mathrm{S} 6$ and better reproducibility of a recently developed analytical method (Molnár et al., 2019), the rhEPO concentration in the elution fractions, the measurement reliability was higher. It was found that the rhEPO purity was around
$90 \%$ in the first elution fraction (peak 1) and $60 \%$ in the second elution fraction (peak 2). The total rhEPO yield was however only slightly below $50 \%$, similarly as for a multimodal anion exchanger in our previous work (Kurák et al., 2019).

\section{Conclusions}

Adsorbent Capto MMC ImpRes has been proven to be a good candidate for rhEPO capture step in rhEPO separation. The most selective binding of rhEPO from a post-cultivation supernatant was 
observed at $\mathrm{pH} 5.5$ and $\mathrm{NaCl}$ concentration of $300 \mathrm{mM}$ in batch equilibrium experiments. Chromatographic runs were used to optimize elution conditions. Two shock-wave gradients, in which $\mathrm{pH}$ was increased to 8 and the $\mathrm{NaCl}$ concentration to $1 \mathrm{M}$, provided the best separation performance. The rhEPO purity of about $80 \%$ was reached when the yield of this target protein was $50 \%$.

\section{Acknowledgments}

This work was supported by grants from the Agency of the Ministry of Education, Science, Research and Sport of the Slovak Republic for the Structural Funds of EU (Grant number: ITMS 26240220071) and the Slovak Research and Development Agency (Grant number: APVV-140474). Dr. Mária Bartošová from the Institute of Viro- logy, Slovak Academy of Sciences, is kindly acknowledged for the preparation of post-culture supernatant of HEK 293.

\section{References}

Adamíková J, Antošová M, Polakovič M (2019) Biotechnol. Lett. 41: 483-493.

Bandi VK, Reddy BRB, Mugthihalli SM, Iyer PK, Pasupuleti P (2017) EP3153522.

Holstein MA, Parimal S, McCallum SA, Cramer SM (2012) Biotechnol. Bioeng. 109: 176-186.

Koticha D, Chaudhari AB (2011) WO2011063195.

Kurák T, Molnár T, Polakovič M (2019) Chem. Pap. 73: 1805-1811.

Molnár T, Bartošová M, Antošová M, Škultéty L, Polakovič M (2019) Chem. Pap. 73: 713-718. 